\title{
Downregulation of cyclooxygenase-1 is involved in gastric mucosal apoptosis via death signaling in portal hypertensive
} rats

\author{
Bin Wu ${ }^{1}$, Lixian Zeng ${ }^{1}$, Ying Lin $^{1}$, Zhuofu Wen ${ }^{1}$, Guihua Chen $^{2}$, Ryuichi Iwakiri ${ }^{3}$, Kazuma Fujimoto ${ }^{3}$ \\ ${ }^{I}$ Department of Gastroenterology, The Third Affiliated Hospital of Sun Yat-Sen University, 600 Tianhe Road, Guangzhou 510630, \\ China: ${ }^{2}$ Transplantation Research Institute, Sun Yat-Sen University, 600 Tianhe Road, Guangzhou 510630, China; ${ }^{3}$ Department of \\ Internal Medicine, Saga Medical School, 5-1-1 Nabeshima, Saga 849-8501, Japan
}

Portal hypertension (PHT) gastropathy is a frequent complication of liver cirrhosis and one of the leading causes of death from cirrhosis. Apoptosis is widely considered to be an active energy-dependent mode of cell death and a distinct entity from necrotic cell death. It is unclear whether gastric mucosal apoptosis is involved in PHT gastropathy. Prostaglandins (PGs) produced through cyclooxygenase (COX) are thought to play a key role in protection of the gastrointestinal mucosa from injury and apoptosis. However, the role of COX in PHT gastropathy is still not clearly understood. The aims of this study were to investigate whether (1) gastric mucosal apoptosis is involved in PHT gastropathy and (2) downregulation of COX contributes to this apoptosis. In this study, we show that gastric mucosal apoptosis was remarkably increased while mucosal proliferation was inhibited in PHT rats. Gastric mucosal COX1 was significantly suppressed at both the mRNA and protein levels, and $\mathrm{PGE}_{2}$ was reduced in $\mathrm{PHT}$ rats. Further, $\mathrm{PGE}_{2}$ treatment suppressed gastric mucosal apoptosis in PHT rats. However, gastric mucosal COX-2 levels did not differ between sham-operated rats and PHT rats. Gastric mucosal levels of tumor necrosis factor- $\alpha$ (TNF- $\alpha$ ) and Fas ligand, but not TNF-related apoptosis-inducing ligand, were increased, and activated caspase-8 and caspase-3 levels were upregulated in PHT rats. The release of cytochrome $\mathbf{c}$ from the mitochondria to the cytosol was not observed in PHT rats. Our data indicate that downregulation of COX-1 is involved in gastric mucosal apoptosis via death signaling-mediated type-I cell death in PHT rats.

Keywords: tumor necrosis factor- $\alpha$, Fas ligand, tumor necrosis factor-related apoptosis-inducing ligand, caspase- 8 , caspase-3 Cell Research (2009) 19:1269-1278. doi: 10.1038/cr.2009.97; published online 11 August 2009

\section{Introduction}

Portal hypertension (PHT) is a frequent complication of liver cirrhosis and one of the leading causes of death from cirrhosis. Clinical and experimental data indicate that in PHT, the gastric mucosa has an increased susceptibility to injury caused by alcohol, nonsteroidal antiinflammatory drugs, ischemia/reperfusion, and impaired mucosal healing [1-4]. It is generally accepted that PHT leads to reduced peripheral vascular resistance, increased

Correspondence: Bin Wu

E-mail: binwu001@hotmail.com

Received 19 January 2009; revised 14 May 2009; accepted 22 May 2009; published online 11 August 2009 regional blood flow, and elevated cardiac output. These hemodynamic events have been collectively referred to as the hyperdynamic circulation [5-7]. Gastric mucosal lesions have been recognized in association with PHT. These lesions, called PHT gastropathy, are now a wellestablished clinical entity [8]. However, the pathogenesis of this disorder is still not clearly understood.

In the development of PHT gastropathy, the role of prostaglandins (PGs) is controversial. Human studies have shown increased, decreased, or unchanged levels of PGs in the gastric mucosa, whereas animal models of PHT gastropathy generally show a reduction of PGs [9-12]. Uniformly, however, a reduction in PGs by inhibitors causes increased gastric mucosal damage in both animal models and patients with PHT $[11,13,14]$. The primary enzyme responsible for PG synthesis, cyclooxygenase 
(COX), exists in at least three isozymes. COX-1 is constitutively expressed in the gastrointestinal tract and most other tissues, whereas COX-2 is expressed at very low levels in most tissues, but has been shown to be induced at the site of inflammation; COX-3 is considered a new and important leader in the generation of anti-inflammatory and analgesic agents [15-17]. It is likely that COX-1 and COX-2 make different contributions to function in different cell types, and they also make different contributions to function in the same cell type under different conditions. Despite the interest in this area, the cellular distributions of COX-1 and COX-2, as well as the relative contributions of COX-1 and COX-2 to mucosal cell apoptosis and proliferation in PHT gastropathy, are largely unknown.

Apoptosis is mediated through a series of biochemical steps, and it has been found that death signaling is involved in apoptosis in a number of cell types. Three death-signaling factors, TNF- $\alpha$, Fas ligand (FasL), and TNF-related apoptosis-inducing ligand (TRAIL), bind to their receptors, resulting in the induction of apoptosis. It is known that two downstream death-signaling pathways mediate apoptosis by the death receptors. Deathsignaling factors, which are ligands for cell surface death receptors, may (1) lead to the clustering of TNF receptor 1 (TNFR1)- or Fas-associated death domain, (2) recruit procaspase- 8 to death-inducing signaling complex, leading to type-I cell death, or (3) induce mitochondrial cytochrome c release, which results in type-II cell death [1821].

In this study, we have examined the role of COX-1 and COX-2 in gastric mucosal apoptosis and proliferation in PHT rats, and investigated whether death signaling was involved in this process. Here, we report that (1) PHT induced gastric mucosal apoptosis and inhibited gastric mucosal proliferation, (2) PHT suppressed COX1 - but not COX-2-derived $\mathrm{PGE}_{2}$ generation in the gastric mucosa, and (3) suppression of COX-1 is involved in gastric mucosal apoptosis via death signaling-mediated type-I cell death in PHT rats.

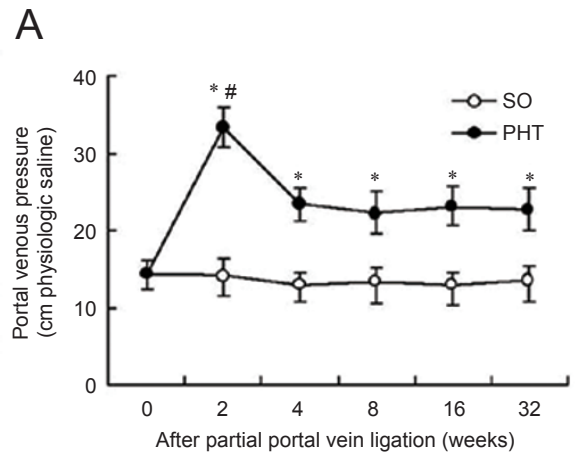

B
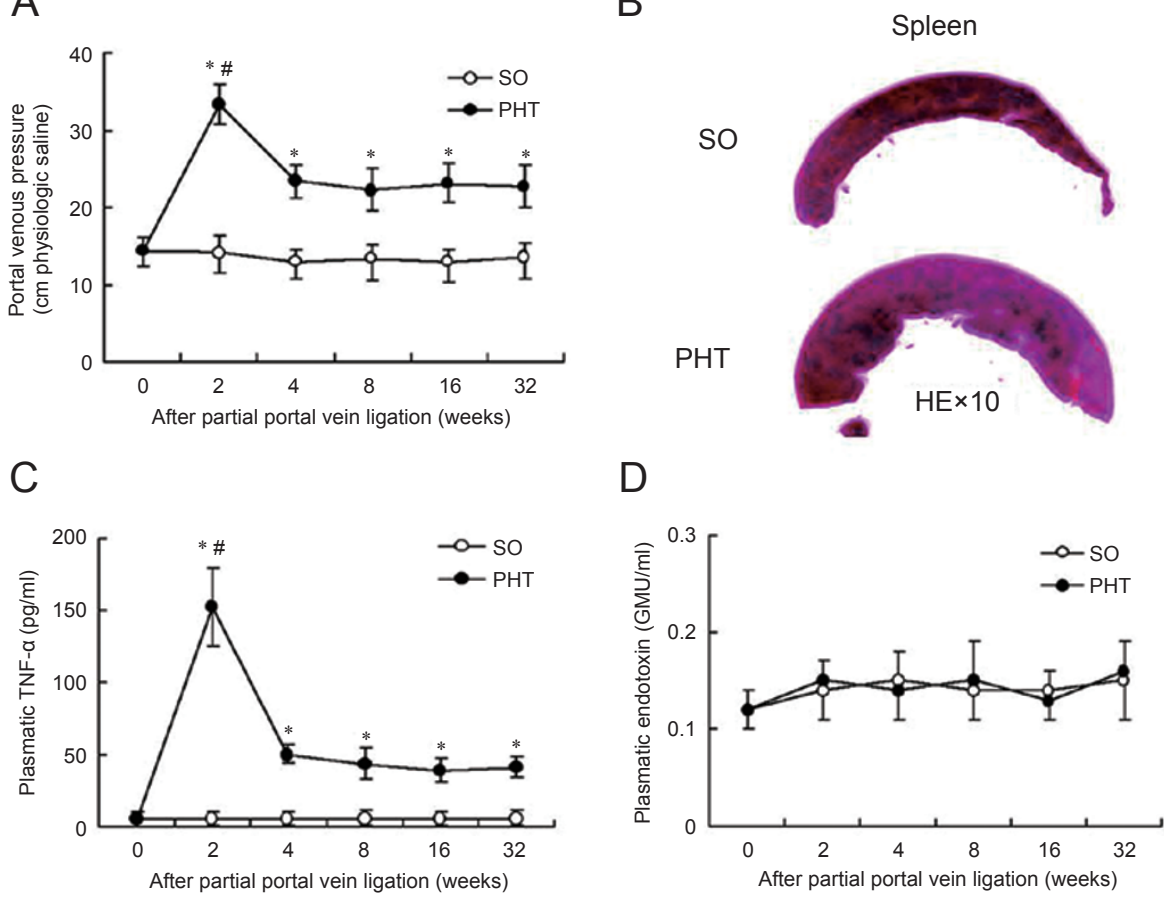

Figure 1 Partial portal vein ligation induced portal hypertension (PHT) and increased plasmatic TNF- $\alpha$ but not endotoxin levels in rats. (A) Partial portal vein ligation significantly increased rat portal venous pressure from 2 weeks to 32 weeks. (B) The spleen showed significant splenemia in PHT rats at 2 weeks. (C) Plasmatic TNF- $\alpha$ levels in PHT rats were significantly increased compared with SO rats from 2 weeks to 32 weeks. (D) Plasmatic endotoxin levels were not significantly different between SO rats and PHT rats from 2 weeks to 32 weeks. Values represent the mean \pm SD, and six rats were studied at each time point in each group. ${ }^{*} P<0.01$, compared with $\mathrm{SO}$ rats. $\# P<0.01$ compared with $4,8,16$, and 32 weeks after partial portal vein ligation. 


\section{Results}

Partial portal vein ligation induced PHT and increased plasmatic TNF- $\alpha$ in rats

After partial portal vein ligation, all rats showed evidence of splanchnic venous congestion with dilation of mesenteric veins and thickening of the mesentery. After 2 weeks of partial portal ligation, the portal venous pressure was significantly higher than that in sham-operated (SO) rats, and the portal venous pressure at 2 weeks was significantly higher than that at $4,8,16$, and 32 weeks in PHT rats (Figure 1A). All PHT rats showed significant congestive splenomegaly. At 2 and 32 weeks, the spleen in PHT rats showed significant splenemia (Figure 1B) and splenitis (data not shown) compared with SO rats. After partial portal ligation, plasma TNF- $\alpha$ levels in PHT rats were significantly higher than in SO rats; additionally, the TNF- $\alpha$ level at 2 weeks was significantly higher than that at 4, 8, 16, and 32 weeks in PHT rats (Figure $1 \mathrm{C})$. In contrast, plasma endotoxin levels were not significantly different between SO and PHT rats (Figure 1D).

PHT induced gastric mucosal apoptosis and inhibited gastric mucosal proliferation

Histological analysis revealed little damage or inflammation in the gastric mucosa of SO rats with ve-

A
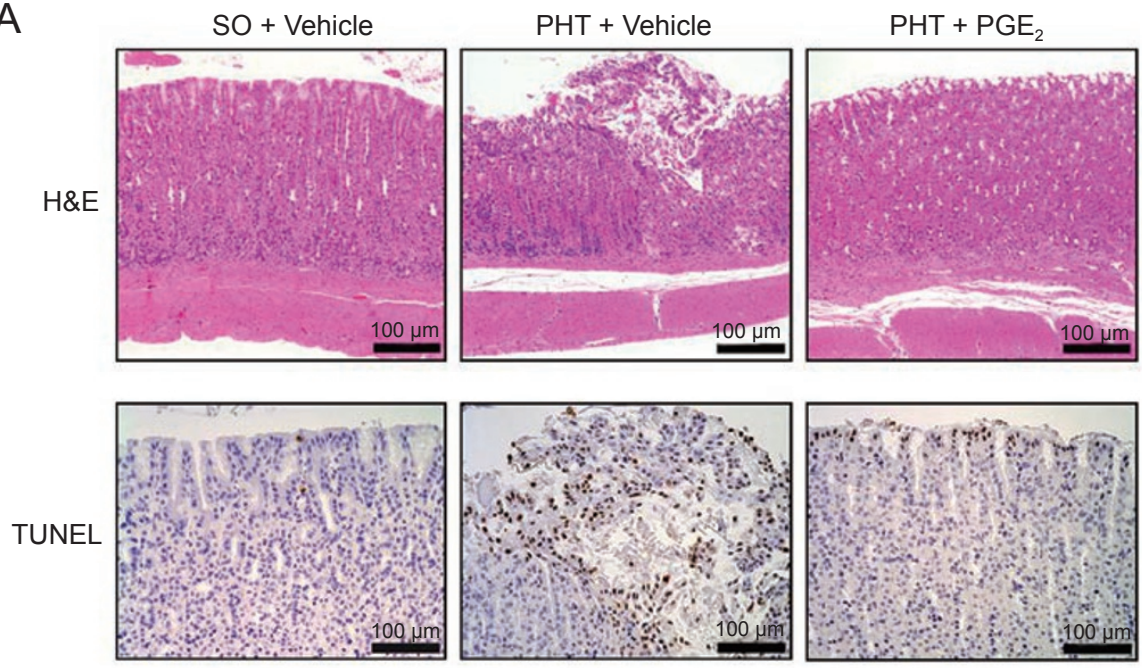

B

C
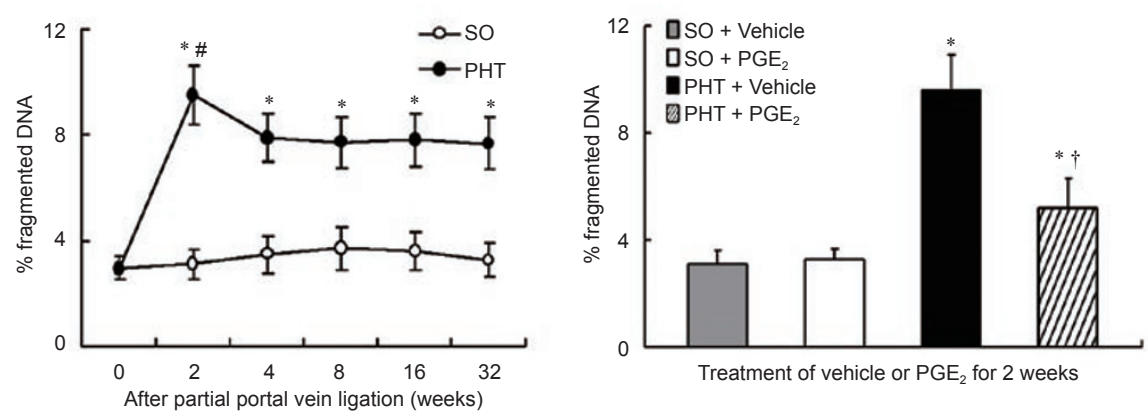

Figure 2 Portal hypertension (PHT) induced gastric mucosal damage and apoptosis. (A) In PHT rats receiving vehicle treatment, a significant inflammatory cell infiltration was observed in the gastric mucosa. Marked mucosal edema with erosion was also found, and the gastric mucosal tight link was destroyed at 2 weeks. Treatment daily with $\mathrm{PGE}_{2}$ for 2 weeks observably suppressed the gastric mucosal inflammatory cell infiltration and mucosal damage. Gastric mucosal TUNEL staining showed that PHT significantly induced gastric mucosal apoptosis, and treatment with $\mathrm{PGE}_{2}$ markedly reduced the gastric mucosal apoptosis at 2 weeks. (B) The gastric mucosal percentage of fragmented DNA was significantly higher in PHT rats compared with $\mathrm{SO}$ rats. (C) $\mathrm{PGE}_{2}$ treatment clearly depressed the percentage of gastric mucosal fragmented DNA in PHT rats, but it did not affect the fragmented DNA in SO rats. Values represent the mean $\pm S D$, and six rats were studied at each time point in each group. ${ }^{*} P<0.01$, compared with $S O$ rats. $\# P<0.01$ compared with $4,8,16$, and 32 weeks after partial portal vein ligation. $\uparrow P<0.01$ compared with $\mathrm{PHT}$ rats receiving vehicle treatment. 
hicle treatment (Figure 2A) or $\mathrm{PGE}_{2}$ treatment (data not shown). After 2 weeks of partial portal ligation, a significant inflammatory cell infiltration affecting the entire gastric mucosa was observed. This inflammation was mainly characterized by infiltration of macrophages, leukocytes, and lymphocytes. Furthermore, a marked mucosal edema with erosion was also found, and the gastric mucosal tight link was destroyed in PHT rats receiving the vehicle treatment (Figure 2A). In contrast, PHT rats receiving $\mathrm{PGE}_{2}$ treatment demonstrated significantly reduced gastric mucosal inflammatory cell infiltration. The mucosal edema was also ameliorated, and gastric mucosal erosion was not observed (Figure 2A).

Terminal deoxnucleotidyl transferase-mediated dUTPbiotin nick end labeling (TUNEL) staining of the gastric mucosa showed that few apoptotic cells were observed at the top of pits in the $\mathrm{SO}$ rats receiving vehicle treatment (Figure $2 \mathrm{~A}$ ) or $\mathrm{PGE}_{2}$ treatment (data not shown). Compared with SO rats receiving vehicle treatment, a significant increase in the number of gastric mucosal apoptotic cells was seen in PHT rats receiving vehicle treatment 2 weeks after partial portal vein ligation (Figure 2A). In PHT rats receiving $\mathrm{PGE}_{2}$ treatment, however, the gastric mucosal apoptotic cells were significantly reduced; apoptotic cells were only observed within the gastric epithelium. The number of apoptotic cells was determined at 36 weeks, and gastric mucosal apoptosis and erosion levels were similar to those seen 2 weeks after partial portal vein ligation (data not shown).

Figure $2 \mathrm{~B}$ shows the ratio of fragmented DNA to total DNA after partial portal vein ligation in the gastric mucosa. At 2 weeks after partial portal vein ligation, the percentage of fragmented DNA $(9.6 \pm 1.1 \%)$ was significantly increased compared with that in SO rats $(3.1 \pm$ $0.5 \%, P<0.05)$. At $4,8,16$, and 32 weeks after partial portal vein ligation, the percentage of gastric mucosal fragmented DNA was lower than that at 2 weeks; however, it was still significantly higher than that in SO rats. To study the effect of PGs on gastric mucosal apoptosis in PHT rats, rats were subcutaneously injected daily with vehicle ( $10 \%$ ethanol in saline) or $\mathrm{PGE}_{2}$ at $3 \mathrm{mg} / \mathrm{kg}$ per day after partial portal vein constriction for 2 weeks. At 2 weeks after partial portal vein ligation, the percentage of gastric mucosal fragmented DNA was investigated; the results are shown in Figure $2 \mathrm{C}$. The percentage of fragmented DNA did not differ significantly between SO rats receiving $\mathrm{PGE}_{2}$ treatment and $\mathrm{SO}$ rats receiving vehicle treatment. However, the percentage of fragmented DNA in PHT rats receiving $\mathrm{PGE}_{2}$ treatment was obviously lower than that in PHT rats receiving vehicle treatment.

Gastric mucosal cellular proliferation was evaluated by assessing proliferating cell nuclear antigen (PCNA).
A

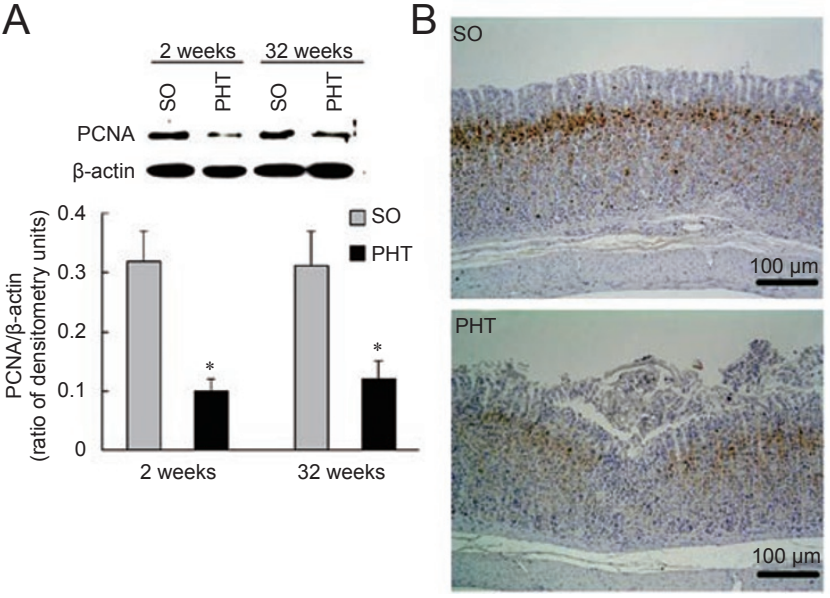

Figure 3 Portal hypertension (PHT) inhibited gastric mucosal proliferation. (A) Western blotting revealed that gastric mucosal PCNA levels were significantly lower in PHT rats than in SO rats. Values represent the mean $\pm S D$, and three rats were used in the study in three separate experiments. ${ }^{*} P<0.01$, compared with SO rats. (B) Immunohistochemical staining for PCNA showed that the number of gastric mucosal PCNA-positive cells in $\mathrm{PHT}$ rats was significantly decreased compared with that in SO rats at 2 weeks.

The result of the western blot is shown in Figure 3A. At 2 and 32 weeks after partial portal vein ligation, the expression of gastric mucosal PCNA was significantly inhibited; quantitative analysis showed that PCNA was reduced by half in comparison with SO rats. Immunohistochemical staining showed that the number of gastric mucosal PCNA-positive cells in PHT rats was significantly lower than that in SO rats in the proliferating cell band (Figure 3B). These results demonstrated that PHT significantly induced gastric mucosal inflammatory response and its consequent damage, increased mucosal apoptosis, and inhibited mucosal proliferation. Treatment of $\mathrm{PGE}_{2}$ markedly alleviated the effect of PHT.

\section{Death signaling mediated gastric mucosal type-I cell death in PHT rats}

We investigated the effect of death signaling on gastric mucosal apoptosis in PHT rats. In SO rats, small amounts of gastric mucosal TNF- $\alpha$, FasL, and TRAIL were detected in the membrane fraction. The amounts of gastric mucosal TNF- $\alpha$, Fas, and FasL, but not TRAIL, were significantly increased in PHT rats compared with SO rats (Figure 4A-4C). Gastric mucosal TNFR1 and DR4 were not significantly different between $\mathrm{SO}$ and PHT rats (Figure 4A and 4C). Little DR5 was detected in either SO or PHT rats (Figure 4C). Two and 32 weeks 


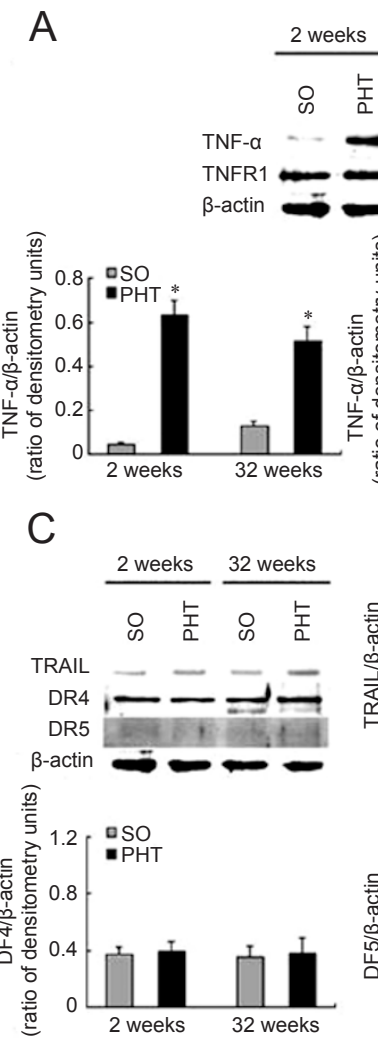

$\mathrm{E}$

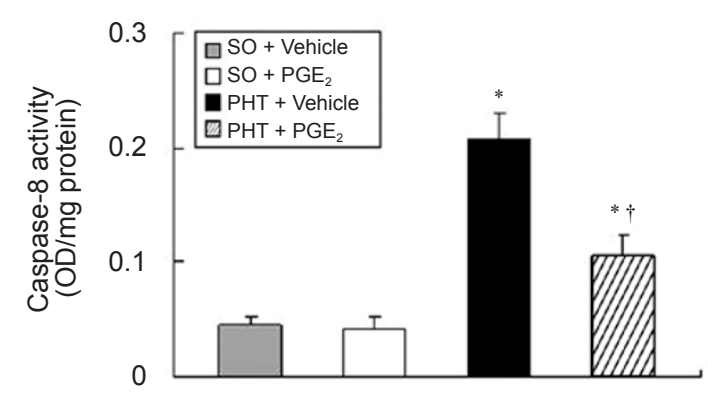

B

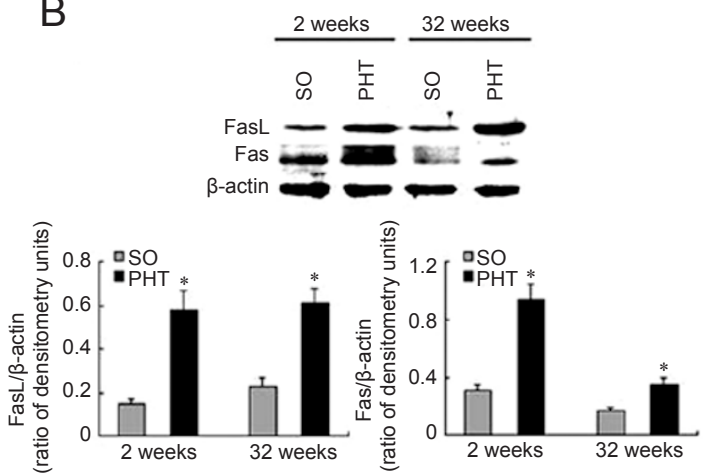

D

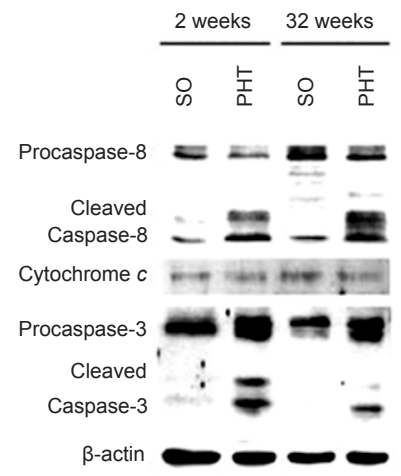

$\mathrm{F}$

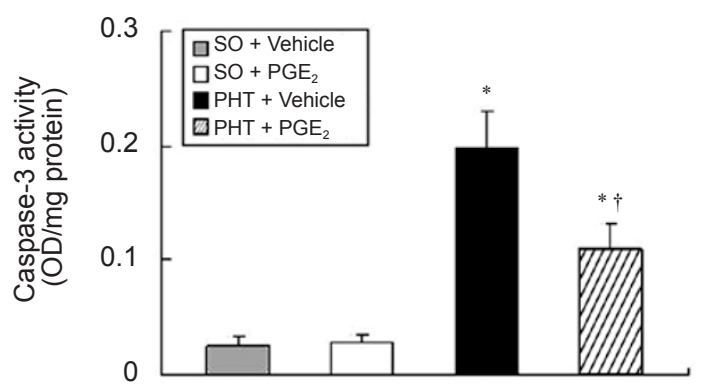

Figure 4 Death signaling mediated gastric mucosal type-I cell death in PHT rats. Equal quantities of protein from the rat gastric mucosa were subjected to immunoblot analysis, the caspase- 8 assay, and the caspase- 3 activity assay. (A) TNF- $\alpha$ and TNFR1. (B) FasL and Fas. (C) TRAIL, DR4, and DR5. (D) Cytosolic caspase-8, cytochrome $c$, and caspase-3. Values represent the mean $\pm S D$, and three rats were used in the study in three separate experiments. $* P<0.01$, compared with $S O$ rats. (E and F) Caspase- 8 and caspase- 3 activities at 2 weeks. Values represent the mean $\pm S D$, and six rats were used in the study in three separate experiments. ${ }^{*} P<0.01$, compared with $\mathrm{SO}$ rats, $\uparrow P<0.01$, compared with $\mathrm{PHT}$ rats receiving vehicle treatment.

after partial portal vein ligation, the expression levels of gastric mucosal-activated caspase- 8 and caspase- 3 were significantly increased in the cytosolic fraction; however, the release of cytochrome $\mathrm{c}$ from the mitochondria to the cytosol was not observed (Figure 4D). Treatment with $\mathrm{PGE}_{2}$ did not affect gastric mucosal caspase- 8 or caspase-3 activity in SO rats, but it significantly suppressed gastric mucosal caspase- 8 and caspase- 3 activity in PHT rats (Figure 4E and 4F). These results suggest that death signaling mediates gastric mucosal type-I cell death in PHT rats.

PHT suppressed COX-1- but not COX-2-derived $P G E_{2}$ generation in the gastric mucosa

After partial portal vein ligation, gastric mucosal $\mathrm{PGE}_{2}$ generation was significantly reduced compared with that 

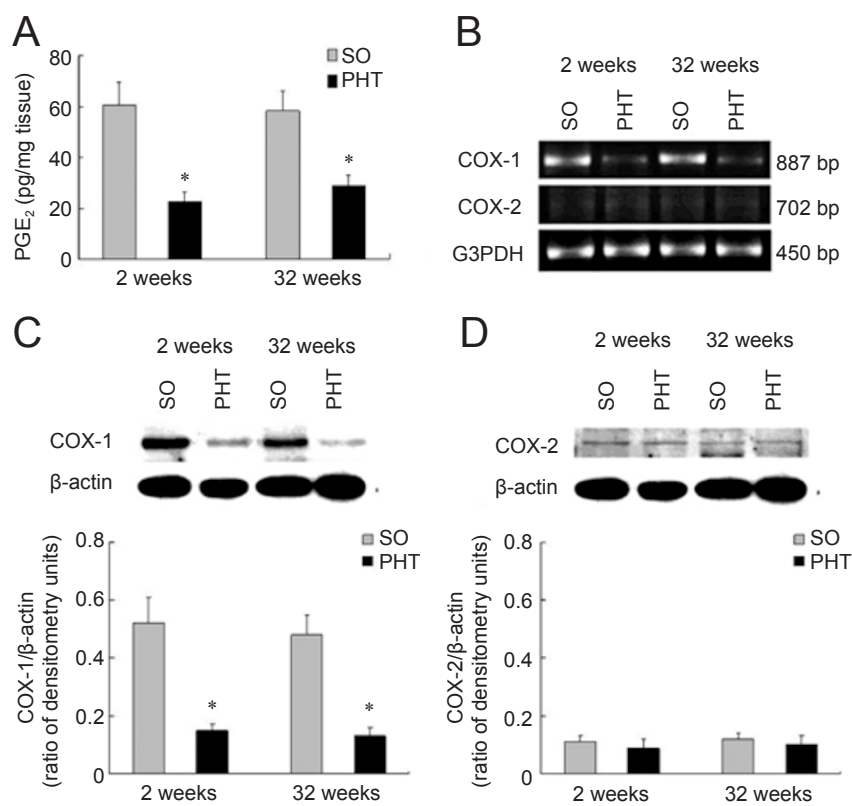

Figure 5 Portal hypertension (PHT) suppressed COX-1- but not COX-2-derived $\mathrm{PGE}_{2}$ generation in the gastric mucosa. (A) Gastric mucosal $\mathrm{PGE}_{2}$ generation was significantly downregulated in PHT rats. Values represent the mean $\pm S D$, and six rats were studied at each time point in each group. $* P<0.01$, compared with SO rats. (B) PHT significantly suppressed COX1 mRNA synthesis but not COX-2 mRNA synthesis. Three rats were used in the study in three separate experiments. (C and D) PHT significantly repressed COX-1 protein levels but not COX-2 protein levels. Values represent the mean $\pm S D$, and three rats were used in the study in three separate experiments. $* P<0.01$, compared with SO rats.

in SO rats by factors of approximately 2.6 and 2 at 2 and 32 weeks, respectively (Figure 5A). Reverse-transcription polymerase chain reaction (RT-PCR) and western blot analyses showed that the synthesis of COX-1 was significantly inhibited in the gastric mucosa compared with SO rats at 2 and 32 weeks after partial portal vein ligation (Figure 5B and 5C). However, the gastric mucosal COX-2 mRNA and protein levels did not differ significantly between $\mathrm{SO}$ and PHT rats (Figure 5B and 5D). These results suggest that PHT suppressed COX1- but not COX-2-derived $\mathrm{PGE}_{2}$ generation in the gastric mucosa.

\section{Discussion}

In this study, we showed that partial portal vein ligation significantly induced splanchnic venous congestion with dilation of mesenteric veins, splenemia, splenitis, and increased portal venous pressure. Furthermore, we found that plasma TNF- $\alpha$ levels, but not plasma endotoxin levels, were significantly increased in PHT rats. The vascular endothelium is considered an autocrine organ that functions using different physiological or pathological signals, among which proinflammatory cytokines (e.g., TNF- $\alpha$ ) play a prominent role [22]. This cytokine stimulates nitric oxide and prostacyclin synthesis, and it may be the stimulus for the production of the two endothelium-derived vasodilators observed in PHT [23]. In fact, recent data suggest that the increased production of TNF- $\alpha$ has a role in vasodilation associated with PHT, and TNF- $\alpha$ has been implicated in the pathogenesis of PHT-associated hyperdynamic circulation [24]. TNF- $\alpha$ increases vascular permeability and causes structural and metabolic changes in vascular endothelial cells. TNF- $\alpha$ also enhances neutrophil attachment to umbilical vein endothelial cells, promoting the inflammatory response to tissue injury. Cirrhotic patients with no signs of infection show increased blood levels of TNF- $\alpha$, and their mononuclear cells exhibit an augmented in vitro synthesis of this cytokine $[25,26]$. Our data show that endotoxin levels do not increase after partial portal vein ligation, suggesting that endotoxin is not involved in the induction of TNF- $\alpha$. However, precisely what induces the increase of TNF- $\alpha$ in PHT remains unclear.

In the current study, our data showed that PHT significantly induced gastric mucosal apoptosis and inhibited mucosal proliferation. It has been found that death signaling is involved in apoptosis in a number of cell types. Three death factors, TNF- $\alpha$, FasL, and TRAIL, bind to their receptors to generate apoptotic signaling [2736]. However, the death factor binding to death receptor can also induce NF- $\kappa \mathrm{B}$ activation via the formation of complex I, resulting in cell survival [27-36]. It is known that two death signal pathways mediate apoptosis via the death receptors TNFR1, Fas, DR4, and DR5 [1821]. Ligand binding of the cell surface death receptors leads to the clustering of the TNFR1- or Fas-associated death domain, and they recruit procaspase- 8 for deathinducing signaling. This signaling leads to type-I cell death or the induction of mitochondrial cytochrome $\mathrm{c}$ release, resulting in type-II cell death. Our results showed that PHT significantly increased cellular TNF- $\alpha$, Fas, and FasL levels but did not increase TRAIL levels. PHT also enhanced the cleavage of caspase- 8 and caspase- 3 , thus increasing the activity of caspase- 8 and caspase- 3 , but it did not induce a release of cytochrome c from the mitochondria to the cytosol in the gastric mucosa. These results indicate that death signaling mediates the gastric mucosal apoptosis via type-I rather than type-II cell death in PHT rats.

Currently, 14 members of the mammalian caspase pro- 
tease family have been cloned and characterized. Several members (caspase- $8,-9$, and -10) contain a large prodomain and are initiators of the apoptotic signaling cascade, whereas other members (caspase-3, -6 , and -7) possess a small prodomain and participate in the execution phase of apoptosis. Our previous studies showed that ischemia/ reperfusion-induced gastrointestinal mucosal apoptosis occurs via the release of cytochrome $c$ from the mitochondria, which then leads to the activation of caspase-9 and caspase- 6 [37-40]. The current data suggest that PHT-induced apoptosis has a different mechanism from that described for ischemia/reperfusion in the gastrointestinal mucosa.

The difference in expression patterns has led to the concept that COX-1 and COX-2 serve different physiological and pathological functions. Thus, it was proposed that COX-1 catalyzes the formation of PGs involved in homeostasis reactions, whereas COX-2-derived PGs function as mediators in pathological reactions such as inflammation and carcinogenesis. Gastric PG synthesis occurs primarily via COX-1. In recent years, many studies have suggested that COX-2 can contribute to the gastric mucosal defense, at least in some circumstances [41, 42]. In this study, our data showed that PHT significantly suppressed gastric mucosal COX-1 and $\mathrm{PGE}_{2}$, but not COX-2. The results suggest that PHT suppresses COX1-derived $\mathrm{PGE}_{2}$ generation in the rat gastric mucosa. To investigate the effect of $\mathrm{PGE}_{2}$ on gastric mucosal damage and apoptosis in PHT rats, the rats were subcutaneously injected daily with vehicle or $\mathrm{PGE}_{2}$ at $3 \mathrm{mg} / \mathrm{kg}$ per day after partial portal vein constriction for 2 weeks. Our results showed that $\mathrm{PGE}_{2}$ treatment significantly suppressed PHT-induced gastric mucosal damage and apoptosis. How COX-derived PGs contribute to mucosal defense remains largely unknown. Our data indicated that inhibition of $\mathrm{COX}$-1-derived $\mathrm{PGE}_{2}$ generation might lead to an increase of death factors in the PHT gastric mucosa, and these death factors may mediate gastric mucosal apoptosis. The current data demonstrated the low expression of COX-2 in the gastric mucosa for both SO and PHT rats. COX-2-derived PGs may represent only a small portion of the total gastric PG pool. In this tissue, the large amount of PGs generated via the COX-1 pathway may create a background that makes it difficult to detect changes in the COX-2 pathway.

In summary, PHT induced gastric mucosal apoptosis and inhibited gastric mucosal proliferation, thereby contributing to PHT gastropathy. PHT suppressed gastric mucosal COX-1 and increased gastric mucosal TNF- $\alpha$, Fas, and FasL levels. This increased death signal leads to caspase- 8 and caspase- 3 activation, resulting in gastric mucosal apoptosis, but does not induce the release of cytochrome $c$ from the mitochondria to the cytosol. Our results suggest that suppression of COX-1 is involved in gastric mucosal damage and apoptosis via death signaling-mediated type-I cell death in PHT rats.

\section{Materials and Methods}

\section{Animals, induction of PHT, and PGE, treatment}

Male Sprague-Dawley rats (200-250 g) were used in this study. The animals were housed in a room illuminated from 8:00 AM to 8:00 PM (12-h light-dark cycle). The rats were allowed access to water and food ad libitum. PHT was induced by a portal vein constriction, as previously described [43]. A laparotomy was performed under halothane anesthesia. Briefly, the portal vein was isolated, and a calibrated constriction was performed using a single ligature of 3-0 silk around the portal vein and a 20-gauge blunttipped needle. The needle was then removed, leaving a calibrated stenosis of the portal vein. This technique involves the initial constriction of the portal vein to approximately $50 \%$ of its diameter. In $\mathrm{SO}$ rats, the same operation was performed with the exception that no ligature was placed after isolating the portal vein. After the operation, the animals were housed in cages and allowed free access to food and water until the experiments. To investigate the effect of PGs on gastric mucosal apoptosis, SO and PHT rats were subcutaneously injected daily with vehicle (10\% ethanol in saline) or $\mathrm{PGE}_{2}$, respectively, at $3 \mathrm{mg} / \mathrm{kg}$ per day for 2 weeks. $\mathrm{PGE}_{2}$ (Cayman Chemical Co., Ann Arbor, MI, USA) was first dissolved in $100 \%$ ethanol and further diluted with saline to reach the desired concentrations (final ethanol concentration 10\%).

\section{Portal venous pressure}

Portal venous pressure was measured before stomach excision. Under halothane anesthesia, a laparotomy was performed and a PE-50 tube was introduced into a peripheral mesenteric vein tributary. With the cava as the reference point, portal venous pressure was measured from the height of the column of physiological saline within the tube. Six rats were studied at each time point in each group.

\section{Determination of plasmatic TNF- $\alpha$ and endotoxin levels}

Blood was withdrawn from the right ventricle when the rat was euthanized. The plasma was collected and kept at $-80{ }^{\circ} \mathrm{C}$ until assayed. Plasmatic TNF- $\alpha$ levels were measured using a rat-specific, commercially available enzyme-linked immunosorbent assay (ELISA) kit (BioSource international, Inc., CA, USA). Results are expressed as $\mathrm{pg} / \mathrm{ml}$. Plasmatic endotoxin was determined using an endotoxin assay kit (HyCult Biotechnology b.v., the Netherlands) according to the manufacturer's directions. IgG was used as a tracer, and the results are expressed as GMU/ml. Six rats were studied at each time point in each group.

\section{Collection of gastric mucosa}

After PHT was induced at 2, 4, 8 16, and 32 weeks, the animals were anesthetized and then euthanized. The entire stomach was carefully removed, placed on ice, and rinsed thoroughly with physiological saline. The stomach was then opened on its lesser curvature longitudinally to expose the gastric mucosa. The mucosal layers were harvested with a glass slide. 


\section{DNA fragmentation assay}

Mucosal scrapings were processed immediately after collection to minimize nonspecific DNA fragmentation. The amount of fragmented DNA was determined as previously described [3740]. Gastric mucosal scrapings were homogenized in a lysis buffer ( $\mathrm{pH}$ 8.0) consisting of $5 \mathrm{mM}$ Tris- $\mathrm{HCl}, 20 \mathrm{mM}$ EDTA (Sigma, St Louis, MO, USA), and 0.5\% (wt/vol) Triton X-100 (Sigma). Aliquots of $1 \mathrm{ml}$ of each sample were centrifuged at $27000 \times \mathrm{g}$ for 20 min at $4{ }^{\circ} \mathrm{C}$ to separate the intact chromatin (pellet) from the fragmented DNA (supernatant). The supernatant was decanted and saved, and the pellet was resuspended in $1 \mathrm{ml}$ of Tris buffer $(\mathrm{pH} \mathrm{8.0)}$ consisting of $10 \mathrm{mM}$ Tris- $\mathrm{HCl}$ and $1 \mathrm{mM}$ EDTA. The pellet and supernatant fractions were assayed for DNA content by using a diphenylamine reaction. Results are expressed as the percentage of fragmented DNA divided by the total DNA. Six rats were studied at each time point in each group.

\section{Histological, immunohistochemical, and TUNEL staining}

Tissue samples were removed from the stomach and spleen, and were immediately fixed in $10 \%$ neutral buffered formalin. The samples were then embedded in paraffin and sectioned. The sections were stained with hematoxylin and eosin (H\&E) and then subjected to histological analysis. PCNA was stained using an ABC staining system (Santa Cruz Biotech, Santa Cruz, CA, USA) with a rabbit polyclonal anti-PCNA antibody (1:400, Santa Cruz Biotech). Fragmented DNA was stained using the TUNEL method, using an Apop Tag Kit (Oncor, Gaithersburg, MD, USA) as previously described [37-40].

\section{Total RNA extraction and RT-PCR}

Total RNA was extracted from the gastric mucosa using ISOGEN (Nippon Gene, Tokyo, Japan) according to the manufacturer's instructions. The RT-PCR product specific for rat COX1 and COX-2 mRNA was amplified using specific primers. The primer pairs and the size of the expected products were as follows (sense and antisense, respectively): COX-1, 5'-AAC CGT GTG TGT GAC TTG CTG AA-3' and 5'-AGA AAG AGC CCC TCA GAG CTC AGT G-3' (887 bp); COX-2, 5'-TGA TGA CTG CCC AAC TCC CAT G-3' and 5'-AAT GTT GAA GGT GTC CGG CAG C-3' (702 bp). For the internal control, expression of the glyceraldehyde 3-phosphate dehydrogenase gene in each sample was also quantified using the primer 5'-TCC ACC ACC CTG TTG CTG TA-3' and the downstream primer 5'-ACC ACA GTC CAT GCC ATC AC-3' (450 bp). RT-PCR was performed on $1 \mu \mathrm{g}$ total RNA using a RT-PCR kit (TOYOBO Co, Osaka, Japan). The PCR products were electrophoresed on $2 \%$ agarose gels and visualized under ultraviolet fluorescent light after staining with ethidium bromide. Three rats were used in the study in three separate experiments.

\section{Purification of proteins and western blot analysis}

The gastric mucosal scrapings were immediately washed twice with ice-cold phosphate-buffered saline ( $\mathrm{pH} 7.4$ ). Total protein, membranous fractions, and cytosolic fractions were purified as previously described [44-46]. PCNA levels were determined in the total protein. TNF- $\alpha$, TNFR1, Fas, FasL, TRAIL, TRAIL receptor 1 (DR4), and TRAIL receptor 1 (DR5) levels were determined in the membranous fraction. Caspase- 8 , cytochrome $c$, and caspase- 3 were analyzed in the cytosolic fraction. Equal quantities of lysates were immersed in a sodium dodecyl sulfate polyacrylamide gel. They were then electroblotted onto a nitrocellulose membrane (Trans-Blot Bio-Rad, Hercules, CA, USA). After blocking with phosphate-buffered saline containing $0.1 \%$ polyoxyethylene sorbitan monolaurate (Tween-20, Sigma) and $5 \%$ skim milk at $4{ }^{\circ} \mathrm{C}$ overnight, the membrane was incubated for $1 \mathrm{~h}$ with the appropriate antibody from the following list: a rabbit polyclonal antiPCNA antibody (1:1 000, Santa Cruz Biotech), a goat polyclonal anti-TNF- $\alpha$ antibody (1:200, Santa Cruz Biotech), a rabbit polyclonal anti-TNFR1 antibody (1:500, Santa Cruz Biotech), a rabbit polyclonal anti-Fas antibody (1:1 000, Santa Cruz Biotech), a rabbit polyclonal anti-FasL antibody (1:500, Santa Cruz Biotech), a rabbit polyclonal anti-TRAIL antibody $(1: 1000$, Santa Cruz Biotech), a mouse monoclonal anti-DR4 antibody (1:500, Santa Cruz Biotech), a goat polyclonal anti-DR5 antibody (1:200, Santa Cruz Biotech), a rabbit polyclonal anti-caspase-8 antibody (1:1 000, Santa Cruz Biotech), a rabbit polyclonal anti-cytochrome c antibody (1:1 000, Santa Cruz Biotech), or a rabbit polyclonal anticaspase-3 antibody (1:1 000, Santa Cruz Biotech). Antigen-antibody complexes were detected with either horseradish peroxidaseconjugated anti-mouse $\mathrm{IgG}$, anti-rabbit $\mathrm{IgG}$, or anti-goat $\operatorname{IgG}(1: 1$ 000, Santa Cruz Biotech). Detection of chemoluminescence was carried out using ECL western blotting detection reagents (Amersham Pharmacia Biotech, UK). Densitometric assessment of the bands from the autoradiogram was performed using Image Gauge VDS (Fujifilm, Tokyo, Japan). Band intensity was quantified by measurement of the absolute integrated optical intensity, which estimates the volume of the band in the lane profile. Three rats were used in the study in three separate experiments. The results are expressed as a ratio to $\beta$-actin densitometry units.

\section{Assay of caspase- 8 and caspase-3 activity}

For analysis of caspase- 8 and caspase- 3 activity, an aliquot of each mucosal scraping sample was placed in $5 \mathrm{ml}$ of ice-cold PBS ( $\mathrm{pH}$ 7.4). After centrifugation (500× $g$ for $10 \mathrm{~min})$, the supernatant was removed and the cell pellet was homogenized in $2 \mathrm{ml}$ of lysis buffer consisting of $50 \mathrm{mM}$ HEPES (pH 7.4), $100 \mathrm{mM} \mathrm{NaCl}, 0.1 \%$ CHAPS, $1 \mathrm{mM}$ DTT, and $0.1 \mathrm{mM}$ EDTA. The lysate was centrifuged at $10000 \times g$ for $10 \mathrm{~min}$ at $4{ }^{\circ} \mathrm{C}$. Aliquots of the supernatant were used to determine protein concentration and caspase- 8 , as well as caspase- 3 activity. Protein concentration was measured using a kit (Bio-Rad). Caspase- 8 and caspase-3 activity was measured using a caspase-8 (Calbiochem, La Jolla, CA, USA) and a caspase-3 (Calbiochem) activity assay kit, respectively, according to the manufacturer's instructions. Six mice in each group were studied. Each experiment was performed in triplicate and repeated at least twice. The results were expressed as absorbance/mg of protein.

\section{Measurement of gastric mucosal $P G E_{2}$}

Gastric mucosal lipids were extracted by homogenizing flashfrozen tissue in cold ethanol/0.1 M sodium phosphate, $\mathrm{pH} 4.0$ $(70 \% / 30 \%, \mathrm{vol} / \mathrm{vol})$, and the mixture was then shaken while incubating at room temperature. An aliquot of the extract was dried under a stream of nitrogen, and the $\mathrm{PGE}_{2}$ concentration was measured using a $\mathrm{PGE}_{2}$-specific ELISA kit (Cayman Chemical) according to the manufacture's directions. The results are expressed as $\mathrm{pg} / \mathrm{mg}$ tissue. Six rats were studied at each time point in each group. 


\section{Statistical analysis}

Results are expressed as mean \pm SD. Data were evaluated by one-way ANOVAs in which multiple comparisons were performed by using the method of least significant difference. Differences were considered statistically significant if the probability of the difference occurring by chance was less than 5 in $100(P<0.05)$.

\section{Acknowledgment}

This work was supported by Grants-in-Aid from the One Hundred Talents Program of Sun Yat-Sen University in China 82000-3171310 (Wu B), and the Ministry of Education, Culture, Sports, Science, and Technology in Japan for Scientific Research 15590656 (Fujimoto K) and 14570478 (Iwakiri R).

\section{References}

1 Kinjo N, Kawanaka H, Akahoshi T, et al. Significance of ERK nitration in portal hypertensive gastropathy and its therapeutic implications. Am J Physiol Gastrointest Liver Physiol 2008; 295:G1016-G1024.

2 Ichikawa Y, Tarnawski A, Sarfeh IJ, Ishikawa T, Shimada H. Distorted microangioarchitecture and impaired angiogenesis in gastric mucosa of portal hypertensive rats. Gastroenterology 1994; 106:702-708.

3 Ishikawa T, Sarfeh IJ, Tarnawski A, Golshan R, Douglas T, Sugiyama M. Epidermal growth factor protects portal hypertensive gastric mucosa in ischemia/reperfusion: the role of capillary endothelia and prostaglandins. Surgery 1992; 112:341-346.

4 Kawanaka H, Tomikawa M, Jones MK, et al. Portal hypertensive gastric mucosa has reduced activation of MAP kinase (ERK2) in response to alcohol injury: a key to impaired healing? FASEB J 2000; 15:574-576.

5 Pateron D, Tazi KA, Sogni P, et al. Role of aortic nitric oxide synthase 3 (eNOS) in the systemic vasodilation of portal hypertension. Gastroenterology 2000; 119:196-200.

6 Yokoyama Y, Baveja R, Sonin N, Clemens MG, Zhang JX. Hepatic neovascularization after partial portal vein ligation: novel mechanism of chronic regulation of blood flow. Am J Physiol Gastrointest Liver Physiol 2001; 280:G21-G31.

7 Yokoyama Y, Wawrzyniak A, Baveja R, Sonin N, Clemens MG, Zhang JX. Altered endothelin receptor expression in prehepatic portal hypertension predisposes the liver to microcirculatory dysfunction in rats. J Hepatol 2001; 35:29-36.

8 McCormack TT, Sims J, Eyre-Brook I, et al. Gastric lesions in portal hypertension: inflammatory gastritis or congestive gastropathy? Gut 1985; 26:1226-1232.

9 Arakawa T, Satoh H, Fukuda T, Nakamura H, Kobayashi K. Endogenous prostaglandin $\mathrm{E}_{2}$ in gastric mucosa of patients with alcoholic cirrhosis and portal hypertension. Gastroenterology 1987; 93:135-140.

10 Wallace JL. Prostaglandins, NSAIDs, and gastric mucosal protection: why doesn't the stomach digest itself? Physiol Rev 2008; 88:1547-1565.

11 Camara PR, Ferraz GJ, Franco-Penteado CF, et al. Ablation of primary afferent neurons by neonatal capsaicin treatment reduces the susceptibility of the portal hypertensive gastric mucosa to ethanol-induced injury in cirrhotic rats. Eur $J$
Pharmacol 2008; 589:245-250.

12 Skill NJ, Theodorakis NG, Wang YN, Wu JM, Redmond EM, Sitzmann JV. Role of cyclooxygenase isoforms in prostacyclin biosynthesis and murine prehepatic portal hypertension. Am J Physiol Gastrointest Liver Physiol 2008; 295:G953G964.

13 Beck PL, Lee SS, McKnight GW, Wallace JL. Characterization of spontaneous and ethanol-induced gastric damage in cirrhotic rats. Gastroenterology 1992; 103:1048-1055.

14 Payen JL, Cales P, Pienkowski P, et al. Weakness of mucosal barrier in portal hypertensive gastropathy of alcoholic cirrhosis. Effects of propranolol and enprostil. J Hepatol 1995; 23:689-696.

15 Chandrasekharan NV, Dai H, Roos KL, et al. COX-3, a cyclooxygenase-1 variant inhibited by acetaminophen and other analgesic/antipyretic drugs: cloning, structure, and expression. Proc Natl Acad Sci USA 2002; 99:13926-13931.

16 Kargman S, Charleson S, Cartwright M, et al. Characterization of Prostaglandin G/H Synthase 1 and 2 in rat, dog, monkey, and human gastrointestinal tracts. Gastroenterology 1996; 111:445-454.

17 Schwab JM, Beiter T, Linder JU, et al. COX-3--a virtual pain target in humans? FASEB J 2003; 17:2174-2175.

18 Chen NJ, Chio II, Lin WJ, et al. Beyond tumor necrosis factor receptor: TRADD signaling in toll-like receptors. Proc Natl Acad Sci USA 2008; 105:12429-12434.

19 Micheau O, Tschopp J. Induction of TNF receptor I-mediated apoptosis via two sequential signaling complexes. Cell 2003; 114:181-190.

20 Scaffidi C, Fulda S, Srinivasan A, et al. Two CD95 (APO-1/ Fas) signaling pathways. EMBO $J$ 1998; 17:1675-1687.

21 Walczak H, Miller RE, Ariail K, et al. Tumoricidal activity of tumor necrosis factor-related apoptosis-inducing ligand in vivo. Nat Med 1999; 5:157-163.

22 Moncada S, Higgs A. The L-arginine-nitric oxide pathway. $N$ Engl J Med 1993; 329:2002-2012.

23 Kaviani A, Ohta M, Itani R, Sander F, Tarnawski AS, Sarfeh IJ. Tumor necrosis factor-alpha regulates inducible nitric oxide synthase gene expression in the portal hypertensive gastric mucosa of the rat. J Gastrointest Surg 1997; 1:371-376.

24 Munoz J, Albillos A, Perez-Paramo M, Rossi I, Alvarez-Mon M. Factors mediating the hemodynamic effects of tumor necrosis factor-alpha in portal hypertensive rats. Am J Physiol Gastrointest Liver Physiol 1999; 276:G687-G693.

25 Deviere J, Content J, Denys C, et al. Excessive in vitro bacterial lipopolysaccharide-induced production of monokines in cirrhosis. Hepatology 1990; 11:628-634.

26 Khoruts A, Stahnke L, McClain CJ, Logan G, Allen JI. Circulating tumor necrosis factor, interleukin-1 and interleukin-6 concentrations in chronic alcoholic patients. Hepatology 1991; 13:267-276.

27 Barnhart $\mathrm{BC}$, Peter ME. The TNF receptor 1: a split personality complex. Cell 2003; 114:148-150.

28 Chaudhary PM, Eby M, Jasmin A, Bookwalter A, Murray J, Hood L. Death receptor 5, a new member of the TNFR family, and DR4 induce FADD-dependent apoptosis and activate the NF-kappaB pathway. Immunity 1997; 7:821-830.

29 Degli-Esposti MA, Smolak PJ, Walczak H, et al. Cloning and characterization of TRAIL-R3, a novel member of the emerg- 
ing TRAIL receptor family. J Exp Med 1997; 186:1165-1170.

30 Emery JG, McDonnell P, Burke MB, et al. Osteoprotegerin is a receptor for the cytotoxic ligand TRAIL. J Biol Chem 1998; 273:14363-14367.

31 Mizoguchi E, Mizoguchi A, Takedatsu H, et al. Role of tumor necrosis factor receptor 2 (TNFR2) in colonic epithelial hyperplasia and chronic intestinal inflammation in mice. Gastroenterology 2002; 122:134-144.

32 Pan G, Ni J, Wei YF, Yu G, Gentz R, Dixit VM. An antagonist decoy receptor and a death domain-containing receptor for TRAIL. Science 1997; 277:815-818.

33 Pan G, O'Rourke K, Chinnaiyan AM, et al. The receptor for the cytotoxic ligand TRAIL. Science 1997; 276:111-113.

34 Schneider $\mathrm{P}$, Thome M, Burns $\mathrm{K}$, et al. TRAIL receptors 1 (DR4) and 2 (DR5) signal FADD-dependent apoptosis and activate NF-kappaB. Immunity 1997; 7:831-836.

35 Walczak H, Degli-Esposti MA, Johnson RS, et al. TRAILR2: a novel apoptosis-mediating receptor for TRAIL. EMBO $J$ 1997; 16:5386-5397.

36 Wallach D, Varfolomeev EE, Malinin NL, Goltsev YV, Kovalenko AV, Boldin MP. Tumor necrosis factor receptor and Fas signaling mechanisms. Annu Rev Immunol 1999; 17:331-367.

37 Wu B, Qiu W, Wang P, et al. p53 independent induction of PUMA mediates intestinal apoptosis in response to ischaemiareperfusion. Gut 2007; 56:645-654.

$38 \mathrm{Wu}$ B, Iwakiri R, Ootani A, Fujise T, Tsunada S, Fujimoto K. Platelet-activating factor promotes mucosal apoptosis via FasL-mediating caspase-9 active pathway in rat small intestine after ischemia-reperfusion. FASEB J 2003; 17:1156-1158.

$39 \mathrm{Wu} \mathrm{B}$, Ootani A, Iwakiri R, et al. Ischemic preconditioning attenuates ischemia-reperfusion-induced mucosal apoptosis by inhibiting mitochondria-dependent pathway in rat small intestine. Am J Physiol Gastrointest Liver Physiol 2004; 286:G580-G587.

$40 \mathrm{Wu} \mathrm{B}$, Iwakiri R, Tsunada S, et al. iNOS enhances rat intestinal apoptosis after ischemia-reperfusion. Free Radic Biol Med 2002; 33:649-658.

41 Fiorucci S, de Lima OM Jr, Mencarelli A, et al. Cyclooxygenase-2-derived lipoxin A4 increases gastric resistance to aspirin-induced damage. Gastroenterology 2002; 123:15981606.

42 Wallace JL, McKnight W, Reuter BK, Vergnolle N. NSAIDinduced gastric damage in rats: requirement for inhibition of both cyclooxygenase 1 and 2. Gastroenterology 2000; 119:706-714.

43 Potenza MA, Botrugno OA, De Salvia MA, et al. Endothelial COX-1 and -2 differentially affect reactivity of MVB in portal hypertensive rats. Am J Physiol Gastrointest Liver Physiol 2002; 283:G587-G594.

44 Ou XW, Wang H, De Plaen IG, Rozenfeld RA, Hsueh W. Neuronal nitric oxide synthase (NOS) regulates the expression of inducible NOS in rat small intestine via modulation of nuclear factor kappa B. FASEB J 2001; 15:439-446.

$45 \mathrm{Wu} \mathrm{D}$, Wallen HD, Nunez G. Interaction and regulation of subcellular localization of CED-4 by CED-9. Science 1997; 275:1126-1129.

46 Yang J, Liu X, Bhalla K, et al. Prevention of apoptosis by Bcl-2: release of cytochrome $c$ from mitochondria blocked. Science 1997; 275:1129-1132. 